\title{
Tuning inflammation in tuberculosis: the role of decoy receptors
}

\author{
Diana Di Liberto, Nadia Caccamo, Serena Meraviglia, Giuliana Guggino, Marco P. La Manna, \\ Guido Sireci, Alfredo Salerno, Francesco Dieli* \\ Dipartimento di Biopatologia e Metodologie Biomediche, Università di Palermo, Corso Tukory 211, Palermo 90134, Italy
}

Received 29 April 2009; accepted 6 May 2009

Available online 18 May 2009

\begin{abstract}
Decoy receptors are "silent scavengers" of CC chemokines and cytokines, which play a key role in damping inflammation and tissue damage. In this review we discuss on recent findings demonstrating that these receptors set the balance between antimicrobial resistance, immune activation and inflammatory response in Mycobacterium tuberculosis infection.
\end{abstract}

(C) 2009 Elsevier Masson SAS. All rights reserved.

Keywords: Decoy receptors; TIR8/SIGIRR; D6; Mycobacterium tuberculosis; Cytokines; Chemokines; Immunopathology; Inflammation

\section{Introduction}

Chemokines (Chemo-attractant Cytokines), acting through their signaling receptors, along with adhesion molecules such as integrins and selectins, selectively co-ordinate the movement of immune cells into and out of specific tissue microenvironments, being an essential component for their recruitment to the infection sites and, consequently, for the arising of the immune response [1].

The large family of chemokines is divided into four subfamilies based on the position of the cysteine residues at their amino terminus: the CC chemokines (CCL1-28) with no aminoacidic residues between the first two cysteines, the CXC chemokines (CXCL1-16) with a single non-conserved aminoacid and the only one $\mathrm{CX}_{3} \mathrm{C}$ chemokine $\left(\mathrm{CX}_{3} \mathrm{CL} 1\right)$ with three aminoacid between them. Finally, the $\mathrm{C}$ chemokines are devoid of the two cysteines characterizing the other chemokines [1].

Furthermore, chemokines can be further divided, according to their expression, into inducible, or inflammatory, and constitutive, or homeostatic: the former are produced in response to different inflammatory stimuli such as infections or tissue damage while the latter are normally expressed, driving leukocyte homing within lymphoid organs. The biological

\footnotetext{
* Corresponding author. Tel: +39 091 6555916; fax: +39 0916555901. E-mail address: dieli@unipa.it (F. Dieli).
}

functions of chemokines, such as chemotaxis, regulation of proliferation, apoptosis, cytokine release, degranulation and Th1/Th2 polarisation, are carried out through the interaction and activation of a distinct subfamily of 18 mammalian structurally related G-protein-coupled signaling receptors, divided into four groups based on the chemokine subclass specificity [1]. Besides the conventional receptors, there exists a small family of atypical and promiscuous receptors sharing a structural similarity with them but referred as "silent chemokine receptors" [2] for their apparent inability to signal upon ligand binding [3].

The lack of signaling functions seems to be due to the altered DRYLAIV motif in the second intracellular loop which, in signaling chemokine receptors, is responsible for G-protein coupling to the receptors; all non conventional receptors are characterized by its absence [4]. Recently, Nibbs and Graham's [3] showed that introducing the DRY motif back into the second intracellular loop restores the signaling function to D6, a decoy receptor for pro-inflammatory chemokines, confirming that this alteration is definitely responsible for the lack of the signaling functions in silent receptors.

\section{Non conventional chemokine receptors}

Three silent chemokine receptors have been identified to date in mammals: DARC (Duffy Antigen Receptor for Chemokines), CCX-CKR and D6 receptors. 
The first is mainly expressed on endothelial cells near to the site of leukocyte extravasation, where it binds mostly CC and CXC pro-inflammatory chemokines [1], and on erythrocytes where it is useful for the entry of some malarial parasites [5].

CCX-CKR binds the constitutive CC chemokines, CCLs $19,21,25$ and weakly, in humans, the follicular CXC chemokine, CXCL13 and probably does not mediate chemotaxis and leukocyte recruitment, being not involved in inflammation [6].

At present, the D6 receptor is the focus of intense investigation in the field of inflammation since it binds many inflammatory CC chemokines (CCLs 2, 3L1, 4, 5, 7, 8, 11-14, 22 and weakly CCL17), without triggering any signals in target cells [7].

Interestingly, many reports identify for D6 a role as a functional decoy receptor that traffics to and from the cell surface to internalize its ligand [8] through recycling endosomes. Once internalized, chemokines are targeted for degradation while the D6 receptor returns to be exposed on the cell surface. The molecular mechanisms of this recycling are still unclear even if it is believed that the D6 receptor is subject to a constitutive ligand-independent phosphorylation [9], helping mediate ligand-independent exocytosis, and that $\beta$-arrestins are not necessary for D6 internalizzation. Conversely, other reports suggest that D6 is not phosphorylated and its constitutive internalization is $\beta$-arrestins-dependent [10].

The D6 receptor is mainly expressed on endothelial cells lining lymphatic channels draining tissues such as skin, gut and syncitiotrophoblast layer of placenta, where it removes inflammatory chemokines, promoting the protection of the immune/inflammatory privilege of the fetus [11].

Furthermore, D6 expression in hematopoietic stem cells (HSC), megakaryocytes, mast cells and DC was supported by the presence of multiple GATA1-binding sites on the D6 promoter. Graham and colleagues [12] have demonstrated that GATA1 is a physiological regulator of D6 expression using a model of mice with a conditional deletion in GATA1, associated to a transcriptional switch-off of D6 expression, in their dendritic cells (DCs).

Interestingly, conversely to data reported in the literature detecting an anti-inflammatory role for D6 owing to its capacity to scavenge circulating pro-inflammatory $\mathrm{CC}$ chemokines, studies in Experimental Autoimmune Encephalomyelitis (EAE), a mouse model of multiple sclerosis, have suggested that D6 functions may be variable and dependent on the setting of the inflammatory conditions. In these EAE model, D6-deficient mice display a significantly lower immune response with a reduced inflammatory leukocyte infiltration in the spinal chord and, consequently, a decreased demyelination [13]. This favourable outcome of the pathology in D6-null mice seems to be associated to a significantly attenuated immune response to the EAE-inducing 'mog' antigen, although the reasons are still unclear.

In contrast, confirming the anti-inflammatory role of D6, its overexpression in human breast cancer cells seems to inhibit proliferation in vitro, tumorigenesis, lung and lymp node metastasis in vivo [14], and to induce a reduction of circulating chemokines, such as CCL2 and CCL5, of vessel density, and tumor-associated macrophage infiltration. Furthermore, D6 expression seems to be positively correlated to disease-free survival rate in cancer patients.

\section{Non conventional cytokine receptors}

Besides chemokines decoy receptors, there exist several cytokine receptors able to generate the interference mechanisms required for a tight regulation of the immune responses [15], such as Toll/IL-1R 8 (Tir8), also known as single Ig IL-1-related receptor (SIGIRR). Tir8 is a member of the IL-1 receptor/Tolllike receptor (TLR) superfamily and an intracellular decoy for components of the signaling pathway. It has a small single extracellular Ig domain, an intracellular TIR domain and a cytosolic tail formed by 95 amino acids [16].

Tir8 has been reported to act as a potent inhibitor of the NF$\kappa \mathrm{B}$ activation by members of the IL-1/TLR family, because its intracellular domain lacks two essential amino acids (Ser447 and Tyr536) in its highly conserved TIR domain, and a trapping agent for TNFR-associated Factor 6 (TRAF6) and IL-1Rassociated kinase 1 (IRAK1) [16]. Thus, Tir8 sequesters the TLR signaling complexes, tuning the action of inflammatory cytokines/chemokines by inhibiting IL-1R and TLR4 signaling [17]. For this reason, Tir8 is referred as the member of the TLR/IL-1R superfamily with unique anti-inflammatory properties.

The Tir8 molecule is expressed on epithelial cell surface and DCs. It has been reported [18] that Tir8-deficient mice show an increased susceptibility to the systemic toxicity of bacterial lipopolysaccharide (LPS) and to dextran sulphate sodium (DSS)-induced intestinal inflammation, proving its crucial role in damping inflammation in the gastrointestinal tract.

More recently, Tir8 has also been reported to negatively regulate in vivo the inflammatory response in infection $[18,19]$.

The role of TLRs in the inflammatory conditions associated to the fungal infection is still unclear. Although inflammation is regarded as crucial in the adaptive immune response towards fungi, its dysregulation may be more harmful for the host than fungal infection itself [20]. Some data provide evidence that Tir8 is required for the host resistance to fungal infection by down-regulating IL-1 signaling-dependent activation of Th17 cell responses, as recently demonstrated for the infection with Candida albicans [21].

Tir8 is known to suppress antimicrobial immunity [20,21] but some recent data identify autoimmunity control as a novel function of this receptor [22]. Tir8 might contribute to the control of autoimmunity suppressing TLR signaling in DCs and autoreactive B cells [23], which are in vitro activated by immune complexes containing the lupus autoantigens U1snRNP or nucleosomes via TLR7 and TLR9, respectively [24,25].

In vivo studies with TLR7 antagonists [26] Tlr7-deficient mice [27] or TLR7 overexpression confirm this concept for 
TLR7 [28] while those performed with TLR9 antagonists and Tlr9-deficient mice are still a matter of debate [29].

Concluding, given their ability to bind pro-inflammatory chemokines and cytokines with no signaling function, the role proposed for "decoy receptor" is to limit the negative and harmful effects of the inflammation caused by the immune responses evoked by self antigens in the autoimmune diseases, by tumors or microorganisms, such as Mycobacterium Tuberculosis ( $M$. tuberculosis), the etiological agent of tuberculosis.

\section{The tight correlation between chemokines, cytokines and granuloma formation in tuberculosis}

The formation of granulomas at the site of mycobacterial infection is an essential component of host immunity for controlling infection. This process is dependent on the activation of mycobacteria-reactive $\mathrm{T}$ lymphocytes [30], particularly IFN $\gamma$-secreting CD4 and CD8 T cells [31]. Granuloma formation, however, is a complex process that requires not only the activation of lymphocytes, but also their recruitment with monocytes to the site of the infection, migration into the tissues, and juxtaposition around mycobacteria-infected macrophages [30]. This colocalization facilitates the activation of bactericidal mechanisms in infected macrophages by $\mathrm{T}$ cellderived cytokines [30]. Some mycobacteria, however, survive within macrophages, and persistent antigenic stimulation perpetuates the process, leading to chronic granuloma formation characterized by dense accumulations of infected macrophages, epithelioid cells, and T lymphocytes [30]. These granulomas contain the mycobacterial infection and prevent dissemination to other organs, but they are also responsible for lung immunopathology, as the granulomas displace and destroy parenchymal tissue [32]. One of the major roles of the granuloma is to localize and contain not only the bacteria but also the inflammatory response to the bacteria itself. Indeed, if immune cells are not tightly controlled within the lungs, this could lead to excess inflammation. Thus, rigorous control of the organization of granulomas is likely necessary to prevent immunopathology. In most cases, after the repair of an inflamed or damaged tissue, inflammation subsides and the tissue returns to its homeostatic norm [33]. However, if the resolution phase of inflammation is ineffective, chronic inflammatory pathologies may develop [33]. Failure to resolve ongoing inflammation is an invariable key feature of pathologies, which are typically characterized by the high-level expression of inflammatory cytokines and chemokines [34]. $\mathrm{TNF} \alpha$ and the related cytokine lymphotoxin [35] are potent pro-inflammatory cytokines with a wide range of activities in both the inflammatory and immune responses, and they play an essential role in host resistance against infection with M. tuberculosis and other mycobacteria [36]. TNF $\alpha$-deficient mice infected by aerosol with $M$. tuberculosis develop normal $\mathrm{T}$ cell responses to mycobacterial antigens, but because of the failure of granuloma formation in the infected organs they are profoundly susceptible to the infection, succumbing with extensive necrosis in the lungs and infected organs [36]. Other than TNF $\alpha$, the role of other soluble mediators in regulating granuloma formation and persistence is poorly understood. Concluding, cytokines, chemokines and their receptors are surely involved in cell migration and are logical candidates for a role in granuloma formation, although their expression has been studied to a limited degree in M. tuberculosis infection [37].

\section{Chemokines and tuberculosis}

A better understanding of the immunological mechanisms of pathogenesis and protection is of essential importance for the design of novel vaccines and immunotherapies against tuberculosis. It has been proposed that the protective response to M. tuberculosis infection requires CD4 and CD8 lymphocytes, the Th1-type cytokines IFN $\gamma$ and TNF $\alpha$, and activated macrophages [38]. The cooperation between the cells and cytokines requires close interaction, which is achieved after migration and granuloma formation in the lungs. The granuloma formation is the hallmark of infection in the lung containing the mycobacteria and creating a microenvironment for immune cell interaction, limiting $M$. tuberculosis growth and dissemination. Chemokines are potent leukocyte activators and chemoattractants aiding granuloma formation and thought to be critical for the immune response to M. tuberculosis [37]. In vitro experiments demonstrated that M. tuberculosis infection of bone marrow-derived mouse macrophages results in the expression of TNF $\alpha$, as well as several chemokines, including ligands for the chemokine receptors CXCR3, CCR5, and CCR2. Neutralization of TNF $\alpha$ by using antibody or TNFR1-deficient macrophages demonstrated that expression of certain chemokines (CXCL9/monokine induced by IFN $\gamma$, CXCL10/IFN-inducible protein 10, CXCL11/IFN-inducible $\mathrm{T}$ cell chemoattractant, CCL5/ RANTES, and CCL2/MCP-1) after M. tuberculosis infection was dependent, at least in part, on TNF $\alpha$. However, the lack of $\mathrm{TNF} \alpha$ did not completely abrogate chemokine expression, indicating that there are other factors, induced as a result of infection, that stimulate chemokine production [39].

In mouse models, gene expression of CXC and CC chemokines has been detected in the lungs after $M$. tuberculosis infection [39]. CXCR3-deficient mice [40] have an impaired granuloma formation after aerosol infection with $M$. tuberculosis, although this effect is transient, occurring at the early stages of infection. CCR2-deficient mice are extraordinarily susceptible to moderate- or high-dose M. tuberculosis administered intravenously [41], and susceptibility is dose-dependent [42]. Conversely, CCR5 [43] may not be essential to the development of a protective response to $M$. tuberculosis infection. CCL2/MCP-1-deficient mice did not demonstrate an increased susceptibility to $M$. tuberculosis infection, but whether cell infiltration or histology was affected in these mice was not reported. However, transgenic mice overexpressing CCL2/MCP-1 were more susceptible to tuberculosis [44].

The CCR5 ligands, CCL3/MIP- $1 \alpha$, CCL4/MIP-1 $\beta$ and CCL5/RANTES, can attract and activate macrophages and Th1 lymphocytes. However studies in $\mathrm{CCR} 5^{-1-}$ mice have found 
that although CCR5 plays a role in the migration of DCs to and from lymph nodes [43], it is not indispensable for granuloma formation and immune protection against $M$. tuberculosis infection. CCL5/RANTES has been associated with the generation of type 1 cytokine-producing granulomas [45]. CCR2-deficient mice are extraordinarily susceptible to moderate- or high-dose $M$. tuberculosis administered intravenously but not to low aerosol doses [42], and CCL2/MCP-1deficient mice do not demonstrate an increased susceptibility to M. tuberculosis infection [46]. However, there is redundancy in the chemokine system. Specific to this study, the chemokines CCL2/MCP-1, CCL3/MIP-1 $\alpha$, CCL4/MIP-1 $\beta$, and CCL5/ RANTES are ligands of CCR5, but CCL3/MIP- $1 \alpha$ and CCL5/ RANTES can also signal through CCR1 and CCR3. This findings make it difficult to dissect the precise roles of individual chemokines and their receptors, suggesting that there are additional factors that must be induced or enhanced to increase the ability of the host to eliminate M. tuberculosis infection.

\section{Role of D6 and Tir8 receptors in the systemic inflammation induced by $M$. tuberculosis infection}

Several studies, reporting no signaling function but only a decoy activity for D6, were mainly addressed to the human molecule [7], although murine D6 owns the same structural features and functions as its human orthologue, acting as a silent receptor and efficient scavenger of inflammatory CC chemokines [47].

To investigate the role of this receptor in the resolution of murine inflammatory processes, different inflammation models were induced in D6-deficient mice $\left(\mathrm{D6}^{-/-}\right)$, such as that locally occurring after subcutaneous injection of Complete Freund's adjuvant (CFA) [47], an emulsion of water in oil composed of inactivated and dried mycobacteria, usually M. tuberculosis, or after repeated applications of 12-O-tetradecanoyl phorbol-13-acetate (TPA), a tumor-promoting agent mutagenizing skin in murine models of cutaneous malignancy [48]. The first model of local inflammation was evaluated by histological analysis of the site of injection at earlier (3 days after CFA injection) and later (21 days after CFA injection) time points. At 3 days after CFA injection, D6 ${ }^{-1-}$ mice were more susceptible to necrosis, angiogenesis and leukocytes infiltration when compared to C57BL/6 normal mice, while at 21 days time point, there were no significant differences between the two groups of mice, both characterized by granuloma-like structures, except for a more intense tissue necrosis found in $\mathrm{D}^{-1-}$ mice.

Moreover, the irritant agent TPA was used to prove the increased susceptibility of $\mathrm{D}^{-1-}$ mice on 2 separate backgrounds $(\mathrm{B} 6 / 129$ and $\mathrm{FVB} / \mathrm{N})$ to develop cutaneous tumors when compared to normal counterparts [48]. Several applications of TPA caused an enhanced inflammation of the skin that was better controlled by normal B6/129 mice than by the corresponding $\mathrm{D}^{-1-}$ mice, showing that D6 limits cytokine bioavailability in the skin, being crucial for the resolution of the inflammatory responses that, in some cases, may be tumorigenic or implicated in carcinoma progression.
In addition, studies of inflammatory responses in Tir8deficient $\left(\mathrm{Tir}^{-/-}\right)$mice [15] have reported on an increased susceptibility to the systemic toxicity of bacterial lipopolysaccharide (LPS), and to intestinal inflammation induced by dextran sulphate sodium (DSS) and colitis-associated cancer [16].

However, very little is known about a possible negative regulatory influence of the D6 and Tir8 molecules in tuning inflammatory responses induced by infection with live microorganisms. This lack of specific knowledge on the biological role of decoy receptors in the resolution of infectioninduced inflammatory responses, prompted us to investigate the role of the D6 and Tir8 receptors in mice infected intranasally with $M$. tuberculosis [49], known to cause a chronic inflammatory response.

The expression levels of the D6 receptor, mainly confined on lymphatic endothelial cells, is not modified in human lungs and lymph nodes of individuals infected with $M$. tuberculosis [49], as well as in lungs, liver and spleen of mice infected with M. tuberculosis infection [49].

D6 ${ }^{-1-}$ mice were found highly susceptible to low intranasal inocula of M. tuberculosis H37Rv (2,000 CFU) with a survival percentage of about $80 \%$ by week 8 and $50 \%$ by week 12 , becoming void at week 16 . Sinilarly, Tir ${ }^{-1-}$ deficient mice succumbed to the same $M$. tuberculosis dose, with a $60 \%$ of mice dying by week 4 and $100 \%$ by week 8 . In both deficient strains however, $M$. tuberculosis CFU loads in the lung, liver and spleen were not different than in receptor proficient wildtype mice, clearly indicating that mortality was not due to failure to control bacterial multiplication and infection.

Histologic analysis revealed severe pulmonary inflammation with edema and necrotic foci as well as diffuse liver and kidney necrosis in D6 ${ }^{-1-}$ mice infected with $M$. tuberculosis (see also Fig. 1), while diffuse severe liver damage was the most important lesion in $\mathrm{Tir}^{-1-}$ mice infected with M. tuberculosis. Supporting these data, M. tuberculosisinfected receptor-deficient mice had elevated levels of after serum transaminases and the renal function was severely compromised with highly increased blood urea nitrogen (BUN) levels and proteinuria. Thus, although their ability to control infecting mycobacteria spreading, $\mathrm{D6}^{-/-}$and $\mathrm{Tir}^{-1-}$ mice have a decreased survival rate associated to lung inflammation, diffuse liver necrosis and renal tissue damage.

\section{7. $\mathrm{D6}^{-/-}$and $\mathrm{Tir}^{-/-}$mice display an exaggerated inflammatory response following $M$. tuberculosis infection}

Lungs and livers of $M$. tuberculosis-infected $\mathrm{D6}^{-/-}$mice were heavily infiltrated by neutrophils, DCs, macrophages, $\mathrm{NK}, \gamma \delta, \mathrm{CD} 4^{+}$and $\mathrm{CD} 8^{+} \mathrm{T}$ cells, when compared to organs of D6 proficient wild-type mice, at all the time points after infection. Moreover, $\mathrm{CD}^{+}$and $\mathrm{CD} 8^{+} \mathrm{T}$ cells infiltrating the lungs of M. tuberculosis infected $\mathrm{D6}^{-1-}$ mice, at 8 and 12 weeks after infection, showed a higher expression of activation markers $\left(\mathrm{CD} 25^{\text {high }}, \mathrm{CD} 44^{\text {high }}, \mathrm{CD} 62 \mathrm{~L}^{\text {low }}\right)$,.

Additionally, higher amounts of macrophages, DCs, CD4 and CD8 T lymphocytes had migrated to mediastinal lymph 

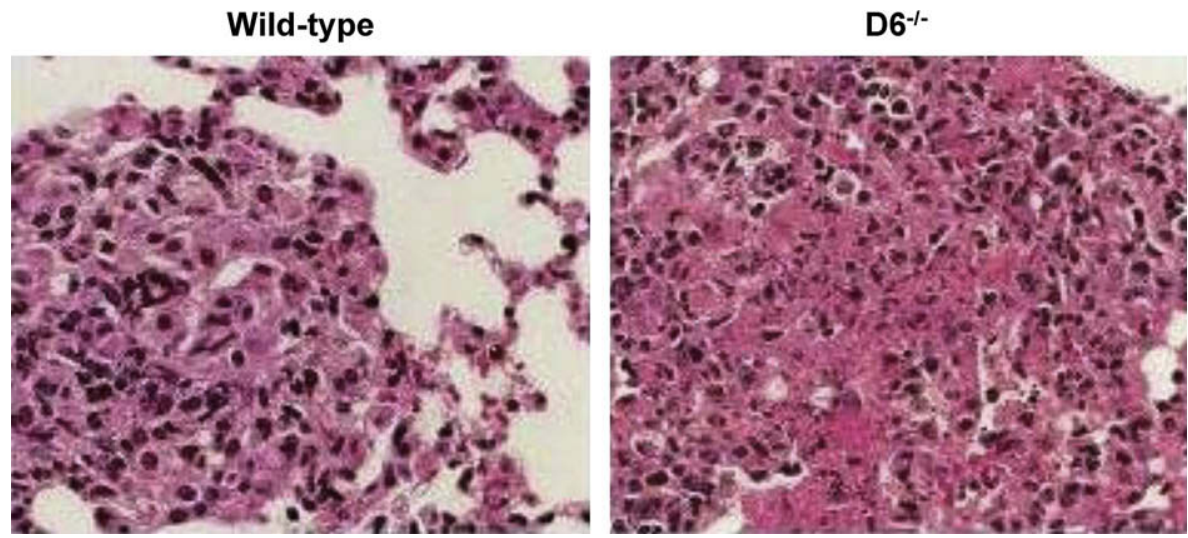

Fig. 1. Lung sections from wild-type and $\mathrm{D}^{-/-}$mice at 12 weeks after infection with $M$. tuberculosis. Hematoxylin and eosin stain at $40 \times$ magnification. Note that in $\mathrm{D6}^{-1-}$ mice the lung space is almost filled with fluid (dark pink staining), infiltrated with predominantly mononuclear cells and few, scattered polymorphonuclear leukocytes cell, and show evidence of cell necrosis and debris. In wild-type mice much more of the lung airspace is intact, with moderate cell infiltration.

nodes of infected $\mathrm{D6}^{-1-}$ mice. The recruitment to lymph nodes caused an increase in the number of specifically primed $\mathrm{T}$ lymphocytes which in turn migrated to lungs, as confirmed by ELISPOT assays performed on lung leukocytes isolated from $\mathrm{D}^{-1-}$ infected mice stimulated in vitro with irradiated BCG infected DCs. In fact, at 8 and 12 weeks after M. tuberculosis infection, the number of IFN $\gamma$ producing $\mathrm{CD} 4^{+}$and $\mathrm{CD} 8^{+} \mathrm{T}$ cells was significantly higher in the lungs of $\mathrm{D}^{-1-}$ mice.

As for $\mathrm{D6}^{-1-}$ mice, the lungs of Tir8 ${ }^{-1-}$ mice were heavily infiltrated by immune cells. However, numbers of DCs, NK, CD4 and CD8 T cells did not differ between Tir8-deficient and -proficient mice at all tested time points after infection, while numbers of neutrophils and macrophages were dramatically increased in Tir8 ${ }^{-l-}$ mice after 2 and 4 weeks of infection.

The increased recruitment of inflammatory cells in different organs of receptor-deficient mice may cause massive, local and systemic production of pro-inflammatory cytokines, such as TNF $\alpha$ and IL- $1 \beta$ and chemokines, which ultimately cause tissue damage, as suggested by date reported in the literature [47,49]. Accordingly, lung mononuclear cells from M. tuberculosisinfected $\mathrm{D}^{-/-}$and Tir8 ${ }^{-1-}$ mice produced larger amounts of these cytokines, and, most important and relevant to the observed phenotype, abundantly higher levels of TNF $\alpha$ and IL-1 $\beta$ were found in bronchoalveolar lavage and sera of M. tuberculosis-infected D6- and Tir-8 deficient mice.

Moreover, the lack of D6 receptor also caused an increase of inflammatory chemokines in blood and inflammed tissues, as demonstrated by the detection of higher CCL2/MCP-1, CCL3/MIP-1 $\alpha$, CCL4/MIP-1 $\beta$ and CCL5/RANTES levels in the bronchoalveolar lavage and serum of $\mathrm{D}^{-1-}$ mice.

To evaluate if the higher levels of circulating pro-inflammatory chemokines were indeed responsible $M$. tuberculosisinfected $\mathrm{D}^{-1-}$ mice were treated with a cocktail of antibodies against the CC chemokines CCL2/MCP-1, CCL3/MIP-1 $\alpha$, CCL4/MIP-1 $\beta$ and CCL5/RANTES or with antibodies against each single chemokine, while $M$. tuberculosis-infected Tir $8^{-1-}$ mice were treated with a mixture of blocking antibodies against $\mathrm{TNF} \alpha$ and IL-1 $\beta$.
As expected, the blockade of all the $\mathrm{CC}$ chemokines caused a remarkable reduction of mortality $\mathrm{D}^{-/-}$infected mice, as showed by only $10 \%$ of mice dying by week 12 and $30 \%$ of mice dying by week 16 . Also Tir8 ${ }^{-1-}$ infected mice treated with neutralizing antibodies to TNF $\alpha$ and IL-1 $\beta$ survived longer, with a death percentage of only $20 \%$ at week 8 and $60 \%$ at week 14 .

Based on these results, we have hypothesized that D6 and Tir8 deficiency induces an increase of pro-inflammatory chemokines/cytokines responsible for the massive and uncontrolled recruitment of immune cells in inflammed sites, exaggerated inflammatory responses and tissue damage.

\section{Concluding remarks}

Better understanding of the immunological mechanisms of pathogenesis and protection are of essential importance for the design of novel vaccines and immunotherapy against tuberculosis. It has been proposed, that the protective response to M. tuberculosis infection, requires CD4 and CD8 lymphocytes, the Th1-type cytokines IFN- $\gamma$ and TNF- $\alpha$, and activated macrophages. The cooperation between the cells and cytokines requires close interaction, which is achieved following migration and granuloma formation in the lungs. The hallmark of infection in the lung is granuloma formation, consisting of clusters of macrophages, lymphocytes and DCs, which physically contains the mycobacteria and creates a microenvironment for immune cell interaction, limiting $M$. tuberculosis growth and dissemination. However, although the production of pro-inflammatory cytokines and chemokines is designed to be protective, if left unchecked, their excessive or inappropriate production may lead to severe inflammatory diseases. Our study indicates an important role for chemokines/cytokines decoy receptors in regulating the extent of the immune response to M. tuberculosis and suggests that decoy receptors, through their ability to avoid excessive production of proinflammatory cytokines or to eliminate circulating chemokines, may prevent excessive tissue damage and overall 
provide a fine mechanism for the control of the balance between protective immune responses and immunopathology.

\section{Acknowledgements}

This study was carried out with financial support from the European Commission, 6 Framework Programme, contract LSHP-CT-2003-503240, "Mucosal Vaccines for PovertyRelated Diseases" (MUVAPRED) and the University of Palermo. We would like to thank Alberto Mantovani, Massimo Locati, Cecilia Garlanda, Annunciata Vecchi, Chiara Buracchi, Manuela Nebuloni, Pere Joan Cardona and Neus Caceres for their helpful advice.

\section{References}

[1] D. Rossi, A. Zlotnik, The biology of chemokines and their receptors, Annu. Rev. Immunol. 18 (2000) 217-242.

[2] A. Mantovani, M. Locati, A. Vecchi, S. Sozzani, P. Allavena, Decoy receptors: a strategy to regulate inflammatory cytokines and chemokines, Trends Immunol. 22 (2001) 328-336.

[3] R. Nibbs, G. Graham, A. Rot, Chemokines on the move: control by the chemokine "interceptors" Duffy blood group antigen and D6, Semin. Immunol. 15 (2003) 287-294.

[4] G.J. Graham, D6 and the atypical chemokine receptor family: novel regulators of immune and inflammatory processes, Eur. J. Immunol. 39 (2009) 342-351.

[5] L.H. Miller, S.J. Mason, J.A. Dvorak, M.H. McGinniss, I.K. Rothman, Erythrocyte receptors for [Plasmodium knowlesi] malaria: Duffy blood group determinants, Science 189 (1975) 561-563.

[6] J.R. Townson, R.J. Nibbs, Characterization of mouse CCX-CKR, a receptor for the lymphocyte-attracting chemokines TECK/mCCL25, SLC/mCCL21 and MIP-3beta/mCCL19: comparison to human CCX-CKR, Eur. J. Immunol. 32 (2002) 1230-1241.

[7] R.J. Nibbs, S.M. Wylie, J. Yang, N.R. Landau, G.J. Graham, Cloning and characterization of a novel promiscuous human beta-chemokine receptor D6, J. Biol. Chem. 272 (1997) 32078-32083.

[8] M. Weber, E. Blair, C.V. Simpson, M. O'Hara, P.E. Blackburn, A. Rot, G.J. Graham, R.J. Nibbs, The chemokine receptor D6 constitutively traffics to and from the cell surface to internalize and degrade chemokines, Mol. Biol. Cell 15 (2004) 2492-2508.

[9] P.E. Blackburn, C.V. Simpson, R.J. Nibbs, M. O'Hara, R. Booth, J. Poulos, N.W. Isaacs, G.J. Graham, Purification and biochemical characterization of the D6 chemokine receptor, Biochem. J. 379 (2004) 263-272.

[10] E. Galliera, V.R. Jala, J.O. Trent, R. Bonecchi, P. Signorelli, R.J. Lefkowitz, A. Mantovani, M. Locati, B. Haribabu, beta-Arrestindependent constitutive internalization of the human chemokine decoy receptor D6, J. Biol. Chem. 279 (2004) 25590-25597.

[11] C. Garlanda, V. Maina, Y. Martinez de la Torre, M. Nebuloni, M. Locati, Inflammatory reaction and implantation: the new entries PTX3 and D6, Placenta 29 (Suppl. B) (2008) 129-134.

[12] C.S. McKimmie, A.R. Fraser, C. Hansell, L. Gutierrez, S. Philipsen, L. Connell, A. Rot, M. Kurowska-Stolarska, P. Carreno, M. Pruenster, C.C. Chu, G. Lombardi, C. Halsey, I.B. McInnes, F.Y. Liew, R.J. Nibbs, G.J. Graham, Hemopoietic cell expression of the chemokine decoy receptor D6 is dynamic and regulated by GATA1, J. Immunol. 181 (2008) 8171-8181.

[13] L. Liu, D. Huang, M. Matsui, T.T. He, T. Hu, J. Demartino, B. Lu, C. Gerard, R.M. Ransohoff, Severe disease, unaltered leukocyte migration, and reduced IFN-gamma production in $\mathrm{CXCR}^{-1-}$ mice with experimental autoimmune encephalomyelitis, J. Immunol. 176 (2006) 4399-4409.

[14] F.Y. Wu, Z.L. Ou, L.Y. Feng, J.M. Luo, L.P. Wang, Z.Z. Shen, Z.M. Shao, Chemokine decoy receptor D6 plays a negative role in human breast cancer, Mol. Cancer Res. 6 (2008) 1276-1288.
[15] A. Mantovani, M. Locati, N. Polentarutti, A. Vecchi, C. Garlanda, Extracellular and intracellular decoys in the tuning of inflammatory cytokines and Toll-like receptors: the new entry TIR8/SIGIRR, J. Leukoc. Biol. 75 (2004) 738-742.

[16] N. Polentarutti, G.P. Rol, M. Muzio, D. Bosisio, M. Camnasio, F. Riva, C. Zoja, A. Benigni, S. Tomasoni, A. Vecchi, C. Garlanda, A. Mantovani, Unique pattern of expression and inhibition of IL-1 signaling by the IL-1 receptor family member TIR8/SIGIRR, Eur. Cytokine Netw. 14 (2003) 211-218.

[17] D. Wald, J. Qin, Z. Zhao, Y. Qian, M. Naramura, L. Tian, J. Towne, J.E. Sims, G.R. Stark, X. Li, SIGIRR, a negative regulator of Toll-like receptor-interleukin 1 receptor signaling, Nat. Immunol. 4 (2003) 920-927.

[18] C. Garlanda, F. Riva, T. Veliz, N. Polentarutti, F. Pasqualini, E. Radaelli, M. Sironi, M. Nebuloni, E.O. Zorini, E. Scanziani, A. Mantovani, Increased susceptibility to colitis-associated cancer of mice lacking TIR8, an inhibitory member of the interleukin-1 receptor family, Cancer Res. 67 (2007) 6017-6021.

[19] C. Garlanda, D. Di Liberto, A. Vecchi, M.P. La Manna, C. Buracchi, N. Caccamo, A. Salerno, F. Dieli, A. Mantovani, Damping excessive inflammation and tissue damage in Mycobacterium tuberculosis infection by Toll IL-1 receptor $8 /$ single Ig IL-1-related receptor, a negative regulator of IL-1/TLR signaling, J. Immunol. 179 (2007) 3119-3125.

[20] L. Romani, P. Puccetti, Controlling pathogenic inflammation to fungi, Expert Rev. Anti Infect. Ther. 5 (2007) 1007-1017.

[21] S. Bozza, T. Zelante, S. Moretti, P. Bonifazi, A. DeLuca, C. D’Angelo, G. Giovannini, C. Garlanda, L. Boon, F. Bistoni, P. Puccetti, A. Mantovani, L. Romani, Lack of Toll IL-1R8 exacerbates Th17 cell responses in fungal infection, J. Immunol. 180 (2008) 4022-4031.

[22] M. Lech, O.P. Kulkarni, S. Pfeiffer, E. Savarese, A. Krug, C. Garlanda, A. Mantovani, H.J. Anders, Tir8/Sigirr prevents murine lupus by suppressing the immunostimulatory effects of lupus autoantigens, J. Exp. Med. 205 (2008) 1879-1888.

[23] A. Marshak-Rothstein, I.R. Rifkin, Immunologically active autoantigens: the role of toll-like receptors in the development of chronic inflammatory disease, Annu. Rev. Immunol. 25 (2007) 419-441.

[24] C.M. Lau, C. Broughton, A.S. Tabor, S. Akira, R.A. Flavell, M.J. Mamula, S.R. Christensen, M.J. Shlomchik, G.A. Viglianti, I.R. Rifkin, A. Marshak-Rothstein, RNA-associated autoantigens activate B cells by combined B cell antigen receptor/Toll-like receptor 7 engagement, J. Exp. Med. 202 (2005) 1171-1177.

[25] M.W. Boule, C. Broughton, F. Mackay, S. Akira, A. Marshak-Rothstein, I.R. Rifkin, Toll-like receptor 9-dependent and -independent dendritic cell activation by chromatin-immunoglobulin G complexes, J. Exp. Med. 199 (2004) 1631-1640.

[26] R.D. Pawar, A. Ramanjaneyulu, O.P. Kulkarni, M. Lech, S. Segerer, H.J. Anders, Inhibition of Toll-like receptor-7 [TLR-7] or TLR-7 plus TLR-9 attenuates glomerulonephritis and lung injury in experimental lupus, J. Am. Soc. Nephrol. 18 (2007) 1721-1731.

[27] S.R. Christensen, J. Shupe, K. Nickerson, M. Kashgarian, R.A. Flavell, M.J. Shlomchik, Toll-like receptor 7 and TLR9 dictate autoantibody specificity and have opposing inflammatory and regulatory roles in a murine model of lupus, Immunity 25 (2006) 417-428.

[28] S. Subramanian, K. Tus, Q.Z. Li, A. Wang, X.H. Tian, J. Zhou, C. Liang, G. Bartov, L.D. McDaniel, X.J. Zhou, R.A. Schultz, E.K. Wakeland, A Tlr7 translocation accelerates systemic autoimmunity in murine lupus, Proc. Natl. Acad. Sci. U.S.A. 103 (2006) 9970-9975.

[29] X. Wu, S.L. Peng, Toll-like receptor 9 signaling protects against murine lupus, Arthritis Rheum. 54 (2006) 336-342.

[30] J.L. Flynn, J. Chan, Immunology of tuberculosis, Annu. Rev. Immunol. 19 (2001) 93-129.

[31] C.G. Feng, A.G. Bean, H. Hooi, H. Briscoe, W.J. Britton, Increase in gamma interferon-secreting $\mathrm{CD} 8(+)$, as well as CD4(+), T cells in lungs following aerosol infection with Mycobacterium tuberculosis, Infect. Immun. 67 (1999) 3242-3247.

[32] I.M. Orme, The immunopathogenesis of tuberculosis: a new working hypothesis, Trends Microbiol. 6 (1998) 94-97.

[33] C. Nathan, Points of control in inflammation, Nature 420 (2002) $846-852$. 
[34] A. Mantovani, The chemokine system: redundancy for robust outputs, Immunol. Today 20 (1999) 254-257.

[35] D.R. Roach, H. Briscoe, B. Saunders, M.P. France, S. Riminton, W.J. Britton, Secreted lymphotoxin-alpha is essential for the control of an intracellular bacterial infection, J. Exp. Med. 193 (2001) 239-246.

[36] A.G. Bean, D.R. Roach, H. Briscoe, M.P. France, H. Korner, J.D. Sedgwick, W.J. Britton, Structural deficiencies in granuloma formation in TNF gene-targeted mice underlie the heightened susceptibility to aerosol Mycobacterium tuberculosis infection, which is not compensated for by lymphotoxin, J. Immunol. 162 (1999) 3504-3511.

[37] H.M. Algood, J. Chan, J.L. Flynn, Chemokines and tuberculosis, Cytokine Growth Factor Rev. 14 (2003) 467-477.

[38] S.H. Kaufmann, How can immunology contribute to the control of tuberculosis? Nat Rev Immunol 1 (2001) 20-30.

[39] H.M. Algood, P.L. Lin, D. Yankura, A. Jones, J. Chan, J.L. Flynn, TNF influences chemokine expression of macrophages in vitro and that of $\mathrm{CD} 11 \mathrm{~b}+$ cells in vivo during Mycobacterium tuberculosis infection, $\mathrm{J}$. Immunol. 172 (2004) 6846-6857.

[40] P. Seiler, P. Aichele, S. Bandermann, A.E. Hauser, B. Lu, N.P. Gerard, C. Gerard, S. Ehlers, H.J. Mollenkopf, S.H. Kaufmann, Early granuloma formation after aerosol Mycobacterium tuberculosis infection is regulated by neutrophils via CXCR3-signaling chemokines, Eur. J. Immunol. 33 (2003) 2676-2686.

[41] W. Peters, H.M. Scott, H.F. Chambers, J.L. Flynn, I.F. Charo, J.D. Ernst, Chemokine receptor 2 serves an early and essential role in resistance to Mycobacterium tuberculosis, Proc. Natl. Acad. Sci. U.S.A. 98 (2001) 7958-7963.

[42] H.M. Scott, J.L. Flynn, Mycobacterium tuberculosis in chemokine receptor 2-deficient mice: influence of dose on disease progression, Infect. Immun. 70 (2002) 5946-5954.
[43] H.M. Algood, J.L. Flynn, CCR5-deficient mice control Mycobacterium tuberculosis infection despite increased pulmonary lymphocytic infiltration, J. Immunol. 173 (2004) 3287-3296.

[44] B.J. Rutledge, H. Rayburn, R. Rosenberg, R.J. North, R.P. Gladue, C.L. Corless, B.J. Rollins, High level monocyte chemoattractant protein1 expression in transgenic mice increases their susceptibility to intracellular pathogens, J. Immunol. 155 (1995) 4838-4843.

[45] S.W. Chensue, K.S. Warmington, E.J. Allenspach, B. Lu, C. Gerard, S.L. Kunkel, N.W. Lukacs, Differential expression and cross-regulatory function of RANTES during mycobacterial (type 1) and schistosomal (type 2) antigen-elicited granulomatous inflammation, J. Immunol. 163 (1999) 165-173.

[46] A. Kipnis, R.J. Basaraba, I.M. Orme, A.M. Cooper, Role of chemokine ligand 2 in the protective response to early murine pulmonary tuberculosis, Immunology 109 (2003) 547-551.

[47] Y. Martinez de la Torre, M. Locati, C. Buracchi, J. Dupor, D.N. Cook, R. Bonecchi, M. Nebuloni, D. Rukavina, L. Vago, A. Vecchi, S.A. Lira, A. Mantovani, Increased inflammation in mice deficient for the chemokine decoy receptor D6, Eur. J. Immunol. 35 (2005) 1342-1346.

[48] R.J. Nibbs, D.S. Gilchrist, V. King, A. Ferra, S. Forrow, K.D. Hunter, G.J. Graham, The atypical chemokine receptor D6 suppresses the development of chemically induced skin tumors, J. Clin. Invest. 117 (2007) $1884-1892$.

[49] D. Di Liberto, M. Locati, N. Caccamo, A. Vecchi, S. Meraviglia, A. Salerno, G. Sireci, M. Nebuloni, N. Caceres, P.J. Cardona, F. Dieli, A. Mantovani, Role of the chemokine decoy receptor D6 in balancing inflammation, immune activation, and antimicrobial resistance in Mycobacterium tuberculosis infection, J. Exp. Med. 205 (2008) 20752084. 